\title{
Pathfinder: Oklahoma's Advanced Traveler Information System
}

\author{
Patrick A. Campbell, John R. Junger, Joseph P. Havlicek, Alan R. Stevenson, and Ronald D. Barnes
}

\begin{abstract}
As part of ITS initiatives worldwide, Advanced Traveler Information Systems (ATIS) provide potential travelers with information and images from roadway and roadside technology. This information benefits the traveling public by improving pre-travel routing and other trip planning capabilities. As part of broader traffic management ITS strategies, these systems improve overall safety by reducing the number of congestion-related crashes and improve the efficiency of transportation systems by increasing roadway capacity. However, the development of an ATIS offers numerous challenges that are unique to the individual region served. The cost of deployment and operation is of critical importance, particularly for states with smaller populations and transportation budgets. This paper presents the design of the Oklahoma Pathfinder ATIS, addressing the state's constraints and exploiting the state's unique ITS deployment. It details the information capabilities, deployment experiences, and traveler reception of the system.
\end{abstract}

\section{INTRODUCTION}

Efforts to utilize the instrumentation of roadways as well as improved technologies in Intelligent Transportation Systems have led to the development of two branches of transportation-stakeholder-driven applications. The first, traffic management, has led to efforts to develop Traffic Management Centers (TMC) throughout the US [1], [2]. These centers are focused on en masse traffic reduction, management of inclement weather resources, police, EMT, and emergency management asset deployment. The second application is Advanced Traveler Information Systems (ATIS) focused on route planning and traffic avoidance on the individual level. Several municipalities have developed independent ATIS systems [3]-[9] and these systems have also been recognized as making significant contributions towards easing overall traffic congestion. While the end users for these two systems differ, ATIS applications aid in the function of broader traffic management and both systems commonly share the same technology: cameras, Remote Traffic Microwave Sensors (RTMS), Dynamic Message Signs (DMS), portable radar systems, dynamic speed limits, etc.

In the development of these two traffic management platforms, the state of Oklahoma has faced several distinguishing challenges. First, the state has the 20th largest area and the 15th lowest population density in the nation [10] resulting in a large number of highway miles per capita. Roughly half

This work was supported in part by grants from the Oklahoma Department of Transportation and the Oklahoma Highway Safety Office.

P. Campbell, J. Junger, J. Havlicek, and R. Barnes are with the University of Oklahoma ITS Laboratory, School of Electrical and Computer Engineering, Norman, OK 73019, USA \{pacampbell, jjunger, joebob, ron\}@ou.edu

A. Stevenson is with the the Oklahoma Department of Transportation, Technology Services Division, Oklahoma City, OK 73105, USA. astevenson@odot.org of the state's 3.7 million person population resides within its two similarly sized metropolitan areas. As one might expect, these urban areas have higher network capacity and higher traffic congestion than the expansive rural areas of the state. Oklahoma's diverse and diffuse system of highways requires that transportation stakeholders are dispersed across the state. Furthermore, these highways and interstates continue to encounter increased traffic each year, while the size of the state's transportation work force is shrinking [11]. As Oklahoma ranks only 43rd in per capita income, it has a need to be much more cost-effective than other relatively wealthier states. At the time of the development of the Oklahoma statewide ITS strategy, the substantial costs associated with the construction and operation of a large, centralized TMC (such as those which exist in practically all other major metropolitan and large scale modern ITS architectures) was prohibitive. In order to serve the needs of the entire state, the decision was made to instead create a decentralized virtual TMC, making the hub of transportation management accessible to traffic and emergency management agencies across the state. This necessitated an investment in a crossstate network architecture, creating a secure, fault tolerant, and ultimately cost saving decentralized TMC system [12]. Into this system, the state has integrated an ATIS [13] capable of using the resources of the decentralized TMC to address the needs of individual travelers on Oklahoma highways.

This paper discusses the development of the Oklahoma Pathfinder ATIS, specifically how the system functions in conjunction with Oklahoma's distributed TMC configuration and how it addresses the state's particular combination of challenges and constraints. The ATIS is an integral component of the state's ITS architecture that serves both the needs of the traveling public and state transportation agents through restricted-access features. It uniquely acts as a bridge between the virtual TMC and third-party data sources. The cost-effective yet powerful technologies used to implement the Oklahoma Pathfinder ATIS can be exploited to improve the design and reduce the cost of development of other ATIS systems. The distinct style of presentation of the Oklahoma Pathfinder ATIS which emphasizes quick dissemination of relevant traffic information is also discussed. This paper further describes the reception of the system and future plans for development.

\section{ATIS AS A COMPONENT OF OKLAHOMA ITS}

The Oklahoma decentralized TMC solution incorporates a private ITS network that forms the core of the Oklahoma ITS architecture. In both sharing the resources of and providing 


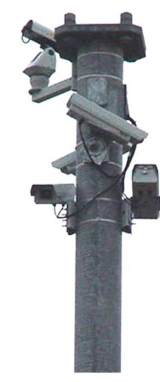

Fig. 1. Camera Pole Configuration.

information for the decentralized TMC, Oklahoma's ATIS has become an integral part of the Oklahoma ITS network.

\section{A. Statewide Architecture}

The Oklahoma ITS network has been designed, developed, and deployed over several years, and has become an effective tool for monitoring and managing traffic conditions. The network consists of a private, and thus secure, dual-ring infrastructure on a fiber-optic backbone [14]. This network implements a distributed architecture that links ITS devices on Oklahoma roadways to controlling stations, called ITS Consoles, located at numerous different stakeholder locations throughout the state [14]. Each ITS Console is capable of monitoring and controlling any ITS device that is visible to it. The network of ITS Consoles as a whole represents a virtual $T M C$, which has offered the state substantial benefits in cost savings, fault tolerance, and scalability [12], [15].

The ITS devices monitored and controlled by this virtual TMC include analog pan/tilt/zoom cameras, digital webcameras, Dynamic Message Signs (DMS's), and Remote Traffic Microwave Sensor (RTMS) speed detectors. In addition, the ITS Consoles are also capable of monitoring device information maintained and provided by third-party vendors.

Most deployed camera locations in Oklahoma consist of a single analog camera and two to four webcameras mounted onto a single pole, as shown in Fig. 1. The analog camera is a pan/tilt/zoom camera which allows ITS Console users to view areas of interest on the highways in great detail, while the webcameras always point in a preset direction, providing a comprehensive overview of traffic conditions at a glance.

\section{B. ATIS as an Extension of the Virtual TMC}

The Oklahoma Pathfinder ATIS is an important component of the virtual TMC. The ATIS website resides on a secure server connected to both the private ITS network and the public internet. This ATIS server forms the important gateway between the Oklahoma ITS network and the public internet. The design of Oklahoma's ATIS is in keeping with the distributed and fault-tolerant strategy of Oklahoma's ITS [14], as the ATIS server has been replicated as a virtual machine which can be instantiated anywhere in the state that ITS network fiber and public internet access coexist. Uniquely, the ATIS server gateway not only provides traffic information from the private network to the general public via the ATIS website, but also provides traffic information from devices on the internet (those not on the private fiber network) to the private ITS Consoles. In addition, a private, passwordprotected interface to the ATIS provides transportation agents across the state with a subset of the information displayed in the virtual TMC, including information that is not displayed on the public interface to the ATIS.

\section{OKLAhoma Pathfinder ATIS OVERVIEW}

Oklahoma's ATIS consolidates many different types of information from several different sources into a concise yet comprehensive view. This information is primarily gathered from both Oklahoma Department of Transportation (ODOT) devices on the private ITS network and from third-party services on the public internet.

\section{A. Information in the ATIS}

Most configuration information about the devices on the private ITS network is communicated to the ATIS server by the ITS virtual TMC consoles. These consoles are designed to operate without a centralized server [14], and as such any console on the network is capable of viewing and configuring all devices available to it. When any console adds, configures, or removes a device, these changes are propagated to the database on the ATIS server. The ATIS website is then able to display these changes as they are made. The remaining traffic management data on the private network is obtained directly by the ATIS server. Since the server is updated with a current list of devices, it directly communicates with those devices to gather additional information that needs to be displayed on the website. This information includes streaming camera feeds and data from ODOT-deployed speed sensors.

In addition, a considerable amount of information displayed on the ATIS website is gathered from third-party services via the public internet. Most of the speed sensors in the Oklahoma City metro area are maintained by Traffic.com, and road construction contractors operating in the state deploy cameras, portable DMS's, and speed sensors within their work zones. These companies provide ODOT with periodic data from their devices. The ATIS server actively fetches this data and combines it with information from the private ITS network for display on the ATIS website. In addition, the information from third-party services gathered by the ATIS server is propagated from the ATIS to each of the ITS virtual TMC consoles on the private network so that the information may be viewed by ITS console users as well.

Not all information gathered by the ATIS server is made available to the general public. For example, RTMS and other speed sensors provide data for each lane on a highway, but these by-lane speeds are not directly displayed for the general public. Therefore, the speed information given to the general public on the ATIS website is summarized with average speeds across all lanes in a given direction. Lane-by-lane speed data, however, may be useful to ODOT engineers who access the virtual TMC through the ATIS website, and so it is desirable to display this data to them. These conflicting requirements are met by providing both a public and private 


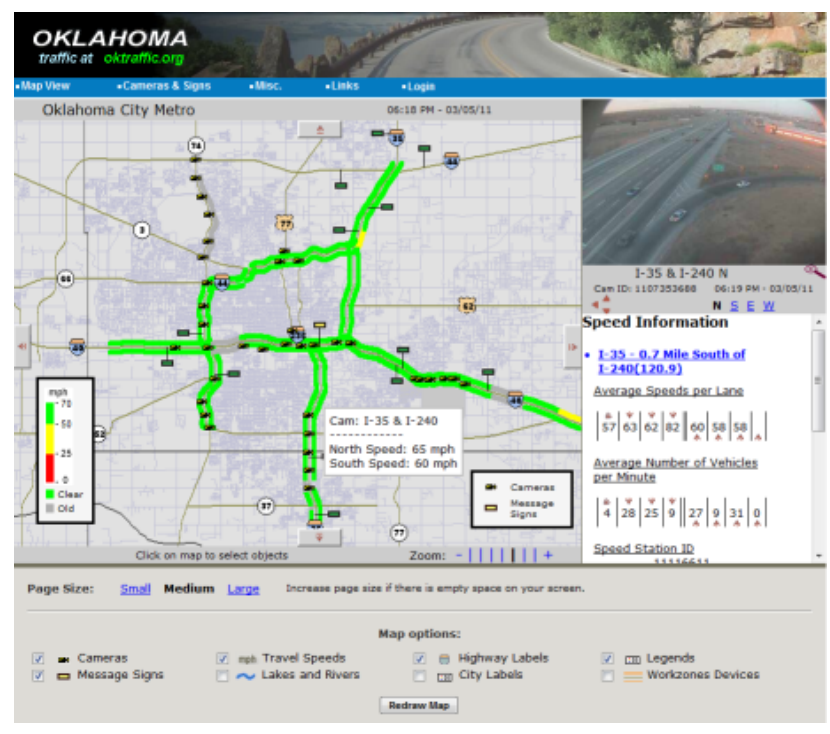

Fig. 2. Oklahoma Pathfinder ATIS Map Page.

interface to the ATIS. The private version requires a password to access and displays all available information and features. The public version appears as an exact copy of the private version with limited access to information and features that are relevant to travelers. This arrangement also provides an important testbed for new website functionality as features that are accessible only on the private interface can be tested by many users before being unlocked on the public interface.

In addition to the speed data mentioned above, a few other features are limited on the public version of the ATIS. One of the most notable is that while camera feeds on the private side show a continuous feed from the camera, the public side displays a ten second captured loop for all cameras. These ten second loops, which are updated every two minutes, reduce bandwidth used by public users. The loops also make it impossible for public users to record continuous streams from the webcams while still allowing travelers to easily see current traffic conditions. Other features which are not available on the public side include a streaming feed from analog cameras, summaries of several Road Weather Information System (RWIS) stations, and summaries of work zone devices.

\section{B. Display of Information on Oklahoma Pathfinder}

There are several components and features of Oklahoma's ATIS system that work together to efficiently present information to the user. Primary among these are map and dedicated information frames located on the main information page of Oklahoma's ATIS, shown in Fig. 2. This page has been designed to allow users to quickly view traffic information at any location with a single mouse click.

The central component of the page in Fig. 2 is a detailed map which by default displays camera devices, dynamic message signs, and highway speeds. At the right side of the map are two dedicated frames that are always visible. The top frame is used to display camera feeds and the bottom frame

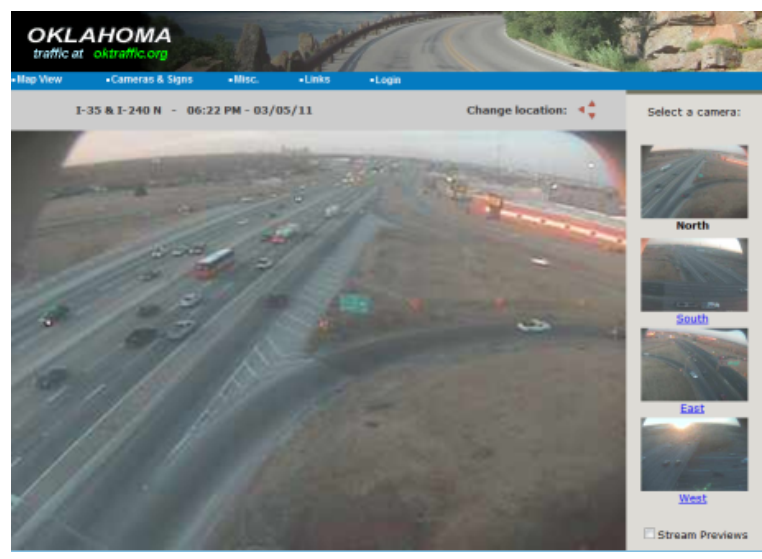

Fig. 3. Oklahoma Pathfinder ATIS Camera View Page.

is used to display text-based information. The information displayed in these dedicated frames is driven by the user's interaction with the map. As the user clicks on the map, the two dedicated frames are updated with information relevant to the selected map location. This framework facilitates the efficient dissemination of information, allowing the user to view relevant information at a glance without requiring navigation around pop-up windows or page redirects.

While the top dedicated frame displays video feeds from cameras selected on the map, the bottom frame is populated when the user clicks on either a Dynamic Message Sign or a location for which highway speeds are available. In the case of a Dynamic Message Sign click, the frame displays the location of the DMS and the message that is currently on the sign. When highway speeds are clicked, the frame displays the location of the speed sensor gathering the speed data, the traffic speed and volume data (including by-lane data through the private interface) and the timestamp at which the speed data was recorded for each lane.

Some of the information described above is also presented at a glance through the use of mouse-over popups that are displayed as the user moves the mouse over the map. In the case of cameras, the mouse-over popup contains the camera name, and in the case of Dynamic Message Signs, the popup contains an image showing the message currently on the sign. When the mouse is moved over a location for which highway speeds are available, the mouse-over popup displays the average speed for each direction of travel on the roadway.

The top dedicated frame can also be used to access a detailed view of a camera feed. Clicking on a video feed within the frame opens a new page, shown in Fig. 3, which displays a larger view of the camera feed to the user as well as preview images from all webcams at the same location. In this page, different webcam feeds may be viewed by clicking one of the preview images or by using the small arrows at the top of the page to move to adjacent camera locations.

The enlarged camera view page, as well as all other pages in the ATIS website, can be accessed by navigating the main menu shown at the top of all informational pages. This menu provides links to several preset map locations as well 


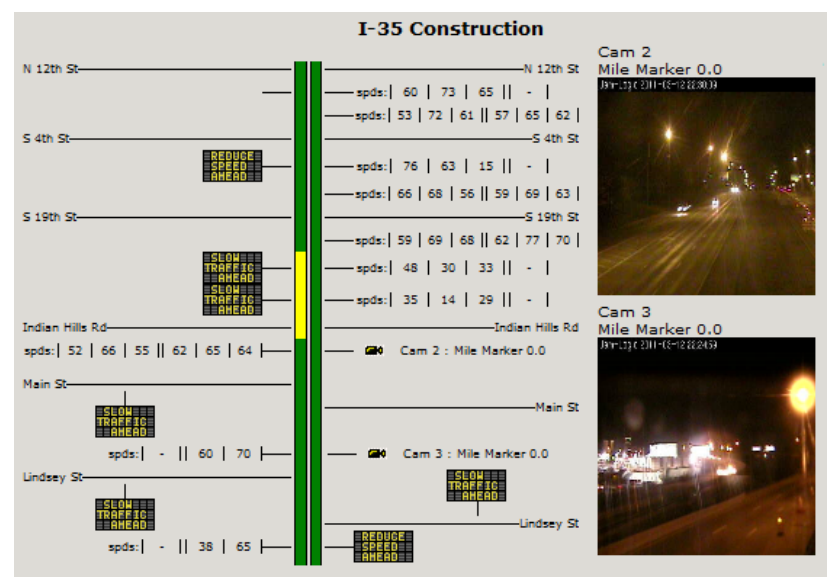

Fig. 4. Oklahoma Pathfinder ATIS Workzone View Page.

as camera view pages for all available camera locations. It also provides links to several other stand-alone pages in the website that are not directly related to the map or camera view pages. In addition, the menu includes links to other useful websites containing traffic and weather information.

\section{Stand-Alone Features}

The Oklahoma Pathfinder ATIS contains several pages containing features that are not directly linked to the other pages in the site. These primarily private interface features often fulfill specific needs of ODOT traffic engineers, assisting them in managing traffic conditions and ITS hardware.

Included in these stand-alone features is a page displaying a live feed from a selected analog camera on the private network. This page connects to a video encoder on the private network which is tuned to a single camera and obtains a video stream from the encoder over Real-time Transport Protocol (RTP). The stream can then be displayed on the client machine using the Quicktime plugin for the user's internet browser. The page is not capable of controlling the analog cameras but does include a control that can select which camera the encoder is tuned to. This allows a transportation or emergency agent on the internet to access the display capabilities of the virtual TMC and view high quality footage from any analog camera on the private network without potentially compromising the secure private ITS network by providing any of the virtual TMC's control capabilities.

Another feature is a public interface Camera Tours page which displays feeds from different webcameras in succession. These sequences of feeds are organized as selectable tours, where each tour is manually configured to contain cameras along a single highway that are facing the same direction. Each camera feed is shown for ten seconds before progressing to the next camera in the tour, allowing users to take a 'virtual drive' down the highway they have selected.

The ATIS website also includes a page that shows information from all roadway devices located in work zones from around the state. The page allows a user to select a single work zone to view, then presents a graphical display showing gathered speeds, DMS messages, and camera images for the work zone. This graphical display, shown in Fig. 4, presents the devices as they are located relative to each other, and offers a quick, comprehensive view of traffic conditions across the entire work zone.

Finally, ODOT also maintains a pilot deployment of RWIS weather stations that are summarized in a dedicated page on the Oklahoma Pathfinder ATIS. These stations report current deck surface, road surface, and sub surface temperatures as well as the surface state (wet, dry, etc.) and provide a camera image for each station. The stations are spread across the state and together provide a partial view of statewide road conditions.

\section{ATIS COMPONENT TECHNOLOGY}

Oklahoma's ATIS employs a variety of technologies and architectures that drive the components and features of the website. This section details two of these technologies which are designed to be cost-effective while enabling the management of several different types of information.

\section{A. Interactive Map}

The maps displayed on the page in Fig. 2 are generated using open source MapScript software [16]. MapScript was developed by the University of Minnesota and generates map images from Geographic Information System (GIS) data.

Resident on the ATIS server are several shape files which contain GIS data for Oklahoma roadways, county lines, bodies of water, and city limits. In order to generate a map image, the ATIS website uses MapScript in conjunction with these shape files to generate an image of Oklahoma roadways and counties for the user's requested latitude, longitude, and zoom level. Based on the map options the user has selected, the generated image can also contain bodies of water, roadway labels, or city outlines. MapScript is then used to include all Oklahoma ITS device and speed information, which is stored in the server's database and continuously updated, in the generated map image. This information is drawn on top of the previously included geographical features, resulting in an image that shows current traffic conditions gathered from the entire Oklahoma ITS network.

In order to reduce server load in cases where several visitors are viewing the website in a short amount of time, a map caching system was also implemented in the Oklahoma Pathfinder ATIS. When a user visits the site and generates a map, a random filename for that map as well as the map latitude, longitude, zoom level, and map options are stored in the server database. If any other user requests the same map image within two minutes, the server directs them to the saved map filename instead of generating a new map image. This results in the possibility of public users viewing traffic conditions that are up to two minutes old, but significantly improves server performance during peak usage times.

The choice of MapScript for Oklahoma's ATIS has several benefits. Primary among these is, of course, the cost of this open source solution. Alternative commercial GIS solutions would be prohibitively expensive. Additionally the unique 
private/public interfaces of the Oklahoma Pathfinder ATIS prohibit even solutions that are free for public websites. A further benefit to using MapScript as a mapping solution is that it is identical to the mapping solution used in the ITS consoles on the Oklahoma private ITS network [17]. Several features that are displayed on the ATIS website are similar to those displayed on the ITS consoles, and a common mapping solution greatly facilitates development on both projects.

The single greatest drawback to the selection of MapScript as a mapping solution is that it is considerably more difficult to use than commercial mapping solutions. ATIS display features can be developed with "cleaner" code that is more quickly implemented using popular mapping tools such as Google Maps or Bing Maps. However, for the Oklahoma Pathfinder ATIS, the cost and compatibility issues mentioned above were enough to justify the implementation using MapScript.

\section{B. Camera Feeds}

Another key technology of Oklahoma's ATIS is an ondemand video streaming architecture developed for the display of camera feeds on the website. This architecture implements camera streaming by obtaining images from available webcams, saving those images on the ATIS server, and redistributing the images to web page visitors. An Open Camera Connection script connects to an available camera, requests a Motion JPEG (MJPG) data stream from the camera over HTTP, and parses the MJPG stream so that individual images are saved on the server. New images from the MJPG stream are saved in a single location, overwriting previous images so that server hard drive space is used efficiently. On a camera feed request, the ATIS server runs the Open Camera Connection script to open a dedicated connection to the requested web camera and stream images from it for fifteen minutes. The server minimizes required bandwidth by preventing more than one dedicated connection at a time to be opened to a single camera.

Once camera images have been obtained by the ATIS server, the images must be delivered to the viewer of the ATIS website. Several potential delivery solutions were examined, most of which involved providing a streaming video format such as QuickTime, RealMedia, MJPG, or Flash. In general however, all of these formats require that the user have a plugin installed on their computer that is capable of processing the video format. As the primary purpose of the Oklahoma Pathfinder ATIS is to disseminate information to the widest possible public audience (including a wide variety of internet access devices), plugin requirement was viewed as a major limitation. Thus, an in-house JavaScript based image delivery solution is used instead.

The JavaScript delivery algorithms used by the Oklahoma Pathfinder ATIS to deliver streaming video images have proven to be very portable. The solution works successfully in all major browsers, operating systems and internet-capable mobile devices including the iPhone and Android phones. In this solution, the user first obtains a camera image from the server. Once that image has loaded, the same image is requested again via JavaScript. As the image on the server is constantly overwritten with newer data, the next image served to the user is a newer frame in the video feed. This also allows the user to dynamically obtain frames at a rate that is controlled by the user's internet connection speed. Thus, on slower connections, such as a mobile device over $3 \mathrm{G}$, images may be shown to the website viewer at a rate of two or three frames per second. On fast ethernet connections images can arrive at a rate of up to twenty frames per second. This delivery method leads to considerable overhead in individual image requests. However, the overhead costs are a trade-off for the portability of the solution.

An additional Loop Capture script was developed to support ten second image loops. This script effectively samples a video stream by establishing a connection to a web camera then copying from the camera image at regular intervals. These copied images are saved to successive loop images which are transmitted to the web page viewer. When a user requests a camera feed through the public interface to the ATIS, if a ten second loop has not been captured for that camera in the previous two minutes, the Loop Capture script is run for the camera. If the same web camera is being viewed on the private version of the ATIS, then the Loop Capture script uses that open connection to perform the loop capture. Otherwise, the Open Camera Connection script is run for fifteen seconds so that a loop capture can be completed. The loop images are delivered to the webpage viewer via the same JavaScript delivery process described above. As new loop images are captured they are immediately transmitted to the user so that playback of the ten second loop begins instantly upon clicking a camera.

\section{RECEPTION AND Future WORK}

Feedback on the Oklahoma Pathfinder ATIS has been generally positive, and the website has seen increasing visitor traffic in the time that it has been online. Recently, the website has also seen exposure in local television news broadcasts.

One of the private interface, stand-alone features of the ATIS is a Site Hits page detailing visitor traffic to the site. This page, shown in Fig. 5, makes use of timelines provided by Google Visualizations [18] to present a quick overview of site usage. The visitor traffic includes numbers for both overall site hits and unique IP addresses that visited the site, where the site hits give an indication of server load and the unique IP addresses give a measure of unique users.

The Site Hits page displays three separate timelines that graph visitor traffic counted at thirty minute, one day, and one week intervals. The thirty minute interval graph is useful for observing fluctuations over a couple of days, while the one day and one week graphs are useful for observing changes in visitor traffic over longer periods. As can be seen in Fig. 5, Oklahoma's ATIS experiences considerable variations in visitor traffic. In a typical day the site sees roughly one to two hundred visitors, with most visitors viewing the site during daylight hours and with slight spikes in traffic in the morning and afternoon hours. Large spikes can be 


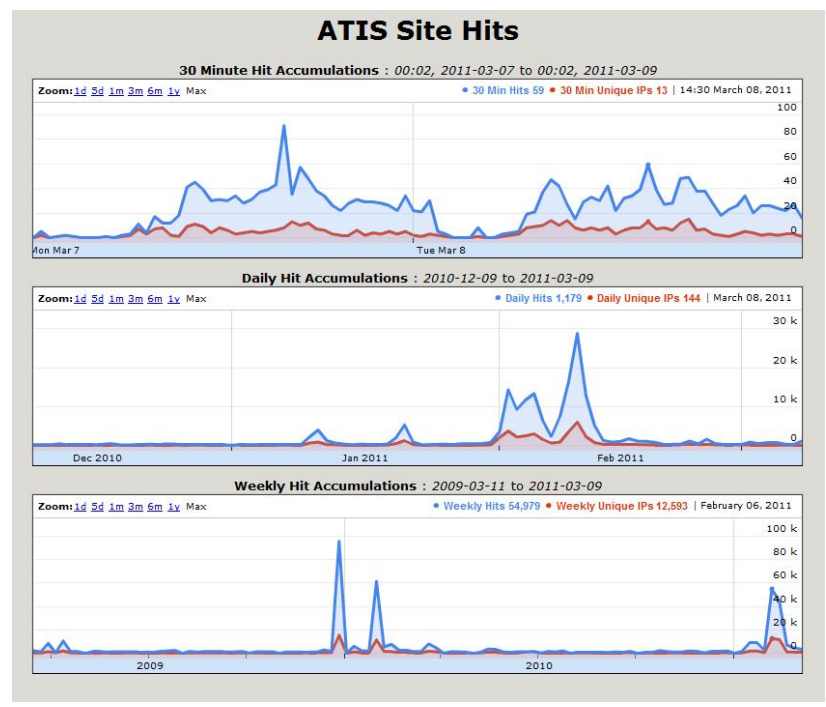

Fig. 5. Oklahoma Pathfinder ATIS Site Hits Page.

seen, however, in the timelines over longer periods, with traffic reaching two to five thousand visitors on some days. These days correspond to time periods during which large ice storms moved through Oklahoma, resulting in a greatly increased demand among the general public for information about traffic conditions.

A contributing factor to the large numbers of site hits during winter storms is the mention of the Oklahoma Pathfinder ATIS in local media at the time of the storms. Local television network websites contained links to the ATIS, which likely led to a larger number of site hits. Since the implementation of streaming camera feeds on the site, local television networks have again mentioned the ATIS during news segments, which has likely resulted in increased site traffic.

Although deployed, Oklahoma's ATIS continues to be actively developed with improvements and new features. One feature under development is a login feature allowing registered public users to retain personalized views of information including previously saved locations on the map or personalized virtual camera tours. Other features being evaluated include the display of traffic incident information on the ATIS and the overlay of real-time NOAA weather radar images. Providing these kinds of information would allow ATIS users to quickly observe and predict changing traffic conditions resulting from collisions and adverse weather conditions.

The Oklahoma Pathfinder ATIS is a unique ATIS implementation that has quickly become a valuable part of the state's traffic management efforts. The website serves the unique and varied traffic information needs of the state with a distinct format that quickly distributes relevant traffic data, including camera video feeds, messages on dynamic message signs, and current highway speeds. The public and private interfaces to the site, combined with the site's rich feature set, simultaneously serve state transportation agents and the general public. As such, Oklahoma's ATIS forms an integral component of the state's ITS architecture that uses cost-effective yet powerful technologies. The exposure the site has seen among the general public, in local media, and among state traffic engineers has led to a desire for continued improvement of the site. Further enhancements to the site will serve to increase its utility while continuing to complement the statewide virtual TMC infrastructure and contribute to the high cost effectiveness of the overall statewide ITS strategy.

\section{REFERENCES}

[1] B. Williams and A. Guin, "Traffic management center use of incident detection algorithms: Findings of a nationwide survey," IEEE Transactions on Intelligent Transportation Systems, vol. 8, no. 2, pp. 351-358, June 2007.

[2] R. Rupert, "The TravTek traffic management center and traffic information network," in Proceedings of the 1991 Vehicle Navigation and Information Systems Conference, Oct. 1991, pp. 757-761.

[3] J. Sweeney, L.E., I. Kamiya, J. Markowitz, J. Georgevich, and M. Crotty, "Travinfo: a progress report," in Proceedings of the 1994 Vehicle Navigation and Information Systems Conference, Aug. 1994, pp. $315-320$.

[4] J. Nelson, F. Spitzer, and S. Stewart, "Experiences gained in implementing an economical, universal motorist information system," in Proceedings of the 1993 IEEE-IEE Vehicle Navigation and Information Systems Conference, Oct. 1993, pp. 67-71.

[5] P. Kumar, V. Singh, and D. Reddy, "Advanced traveler information system for Hyderabad City," IEEE Transactions on Intelligent Transportation Systems, vol. 6, no. 1, pp. 26-37, March 2005.

[6] H. Okamoto, "Recent developments in Japanese traffic information supply system," in Proceedings of the 1993 IEEE-IEE Vehicle Navigation and Information Systems Conference, Oct. 1993, pp. 204-207.

[7] T. Geehan and L. Suen, "User acceptance of advanced traveler information systems for elderly and disabled travellers in Canada," in Proceedings of the 1993 IEEE-IEE Vehicle Navigation and Information Systems Conference, Oct. 1993, pp. 389-392.

[8] M. Hu, Y. Wang, and Q. Shi, "Developing Beijing traveler information systems framework," in Proceedings of 5th IEEE International Conference on Intelligent Transportation Systems, Sept. 2002, pp. 381-386.

[9] Y. Ma, J. Lu, Q. Xiang, H. Zhao, and J. Xie, "Planning of highway traveler information system of Jiangsu Province in China," in Proceedings of the 10th International IEEE Conference on Intelligent Transportation Systems, Oct. 2007, pp. 247-252.

[10] U.S. Census Bureau. Census bureau home page. Visited March, 2011. [Online]. Available: http://www.census.gov/

[11] "ODOT system status report," Oklahoma Department of Transportation, Tech. Rep., June 2005. [Online]. Available: http://www.okladot.state.ok.us/public-info/civic/pdfs/systemstatus.pdf

[12] B. Kilani, E. Vorakitolan, J. Havlicek, M. Tull, and A. Stevenson, "Distributed ITS control and the Oklahoma virtual TMC," in Proceedings of the 12th International IEEE Conference on Intelligent Transportation Systems, Oct. 2009, pp. 785-790.

[13] Oklahoma Pathfinder ATIS. Visited March, 2011. [Online]. Available: http://www.oktraffic.org/

[14] R. Huck, J. Havlicek, J. Sluss, Jr., and A. Stevenson, "A low-cost distributed control architecture for intelligent transportation systems deployment in the State of Oklahoma," in Proceedings of the 8th International IEEE Conference on Intelligent Transoprtation Systems, Vienna, Austria, Sept. 2005, pp. 919-924.

[15] B. Kilani, "Statewide console for distributed control of intelligent transportation systems," Master's thesis, The University of Oklahoma, 2010.

[16] MapTools.org. PHP MapScript. Visited March, 2011. [Online]. Available: http://www.maptools.org/php_mapscript/

[17] P. A. Campbell, "Designing the Oklahoma Statewide ITS around free and open source software," Presented at the 12th International IEEE Conference on Intelligent Transportation Systems, October 2009.

[18] Google.com. Google visualization API gallery. Visited March, 2011. [Online]. Available: http://code.google.com/apis/visualization/ documentation/gallery.html 